\title{
Lunin-Maldacena backgrounds from the classical Yang-Baxter equation - towards the gravity/CYBE correspondence
}

\author{
Takuya Matsumoto $^{a}$ and Kentaroh Yoshida ${ }^{b}$ \\ ${ }^{a}$ Institute for Theoretical Physics and Spinoza Institute, Utrecht University, \\ Leuvenlaan 4, 3854 CE Utrecht, The Netherlands \\ ${ }^{b}$ Department of Physics, Kyoto University, \\ Kyoto 606-8502, Japan \\ E-mail: t.matsumoto@uu.nl, kyoshida@gauge.scphys.kyoto-u.ac.jp
}

ABSTRACT: We consider $\gamma$-deformations of the $\mathrm{AdS}_{5} \times \mathrm{S}^{5}$ superstring as Yang-Baxter sigma models with classical $r$-matrices satisfying the classical Yang-Baxter equation (CYBE). An essential point is that the classical $r$-matrices are composed of Cartan generators only and then generate abelian twists. We present examples of the $r$-matrices that lead to real $\gamma$-deformations of the $\mathrm{AdS}_{5} \times \mathrm{S}^{5}$ superstring. Finally we discuss a possible classification of integrable deformations and the corresponding gravity solution in terms of solutions of CYBE. This classification may be called the gravity/CYBE correspondence.

KEywords: AdS-CFT Correspondence, Integrable Field Theories, Sigma Models ArXiv EPRINT: 1404.1838 


\section{Contents}

1 Introduction 1

2 Abelian twists of the $\mathbf{A d S}_{5} \times \mathrm{S}^{5}$ superstring 3

2.1 Deformed $\mathrm{AdS}_{5} \times \mathrm{S}^{5}$ string actions with $\mathrm{CYBE} \quad 3$

2.2 Classical R-operators for abelian twists 4

$3 \gamma$-deformed $\mathrm{AdS}_{5} \times \mathrm{S}^{5}$ from classical $r$-matrix $\quad 4$

$3.1 \gamma$-deformed $\mathrm{AdS}_{5} \times \mathrm{S}^{5}$ with three parameters 4

3.2 One-parameter case 5

$\begin{array}{lll}3.3 & \text { Three-parameter case } & 6\end{array}$

4 Conclusion and discussion $\quad 8$

$\begin{array}{lr}\text { A Our notation and convention } & 8\end{array}$

$\begin{array}{ll}\text { B Rewriting } \gamma \text {-deformed backgrounds } & 9\end{array}$

$\begin{array}{ll}\text { C Derivation of deformed actions } & 10\end{array}$

\section{Introduction}

The AdS/CFT correspondence [1-3] has been well investigated and there would be no doubt for its validity at least in the planar limit. However, it is still important to consider the fundamental structure of the duality in order to make our understanding much deeper and look for a clue of new physics. The discovery of the integrable structure behind it [4] would play an important role along this direction. The integrability provides a guiding principle to extend the AdS/CFT correspondence by elaborating integrable deformations of it.

Our concern here is the integrable structure of type IIB superstring on $\operatorname{AdS}_{5} \times \mathrm{S}^{5}$. The Green-Schwarz string action is constructed from the following supercoset [5]:

$$
\operatorname{PSU}(2,2 \mid 4) /[\mathrm{SO}(1,4) \times \mathrm{SO}(5)] .
$$

The $\mathbb{Z}_{4}$-grading of this coset ensures the classical integrability [6]. For similar argument based on another coset representation [7], see [8,9]. Possible supercosets, which lead to classically integrable and consistent string theories, are classified in [10, 11].

A recent interest is to consider $q$-deformations of the $\mathrm{AdS}_{5} \times \mathrm{S}^{5}$ superstring. There are two kinds of $q$-deformations, 1) standard $q$-deformations [12-14] and 2) non-standard $q$ deformations (also called Jordanian deformations) $[15,16]$. Both of them are based on the Yang-Baxter sigma model approach proposed by Klimcik [17-19], ${ }^{1}$ where linear R-operators

\footnotetext{
${ }^{1}$ For quantum aspects of Yang-Baxter sigma models, see [20].
} 
constructed from classical $r$-matrices play the central role in constructing deformed classical actions. As a characteristic property, the former is based on the modified Yang-Baxter equation (mCYBE) and the latter is on the classical Yang-Baxter equation (CYBE).

For standard $q$-deformations of sigma models, many works have been done so far. Although deformed target spaces are not represented by symmetric cosets ${ }^{2}$ and there is no general prescription to argue the integrability, many techniques have been developed and various aspects have been revealed. Especially for squashed $S^{3}$, the Lax pair was presented in [22] and the classical integrable structure has been elaborated in the subsequent works [23-34]. As a possible way toward higher-dimensional cases, the Yang-Baxter sigma model approach was proposed by Klimcik [17-19]. Though it was originally argued for principal chiral models, Delduc, Magro and Vicedo succeeded to generalize it to symmetric coset cases [35], where the standard $q$-deformed algebra is presented as a generalization of $[27,28]$. Then they have constructed a standard $q$-deformed $\mathrm{AdS}_{5} \times \mathrm{S}^{5}$ superstring action with a linear R-operator satisfying mCYBE [36]. The coordinate system was introduced and the metric in the string frame and NS-NS two-form have been determined so far [37]. However the full solution has not been obtained yet in type IIB supergravity. For further discussion with specific values of the deformation parameter, see [38]. A related mirror TBA is also discussed in [39]. It would be an important task to compare the results with the deformed S-matrices [40-47].

For non-standard $q$-deformations, deformed $\mathrm{AdS}_{5} \times \mathrm{S}^{5}$ superstring actions have been constructed with linear R-operators satisfying CYBE [48]. A remarkable point is that partial deformations are possible in comparison to the standard q-deformation. For a simple example of the classical $r$-matrices deforming only $\mathrm{AdS}_{5}$, the metric and NS-NS two-form are obtained by a coset construction with an appropriate coordinate system. Then the complete type IIB gravitational solution has been found [49]. In particular, the solution is real and there is no curvature singularity, while the tidal force diverges at the boundary except for a specific surface. It also contains the three-dimensional Schrödinger spacetime as a subspace and, for the subsector analysis, one can use the results obtained in a series of works [50-53]. All of the results are consistent to the recent analysis [54].

In this note, we consider $\gamma$-deformations of the $\mathrm{AdS}_{5} \times \mathrm{S}^{5}$ superstring as Yang-Baxter sigma models with classical $r$-matrices satisfying CYBE. An essential point is that the classical $r$-matrices are composed of Cartan generators only and do not satisfy the nilpotency condition in comparison to Jordanian deformations considered in [48]. These generate abelian twists which are particular examples of the Drinfeld-Reshetikhin twists $[12,13,55]$. We present examples of the $r$-matrices that lead to real $\gamma$-deformations of the $\operatorname{AdS}_{5} \times \mathrm{S}^{5}$ superstring. Our analysis is concerned with the metric and NS-NS two-form only. The coincidence gives a strong evidence in favor of the equivalence of the Yang-Baxter sigma models and the gravity solutions. For the definiteness, it is also necessary to show the coincidence of the R-R fields. However, it would be very complicated to extract them from the fermionic sector of the Yang-Baxter sigma models with the supercoset construction. It is an important issue in the future. Finally we discuss a possible classification of integrable

\footnotetext{
${ }^{2}$ For some examples of non-symmetric cosets, see [21].
} 
deformations and the corresponding gravity solution in terms of solutions of CYBE. This classification may be called the gravity/CYBE correspondence.

This note is organized as follows. In section 2 we introduce skew-symmetric classical $\mathrm{R}$-operators composed of Cartan generators only. These are solutions of CYBE, but do not satisfy the nilpotency condition. With the R-operators, we present classically integrable and $\kappa$-invariant string actions. Section 3 presents simple examples. We show a relation between a classical $r$-matrix and a TsT transformation which leads to a real $\gamma$-deformed $\mathrm{AdS}_{5} \times \mathrm{S}^{5}$. It is straightforward to generalize the classical $r$-matrix for three-parameter $\gamma$-deformations. As a result, the Lunin-Maldacena background is contained as a particular case. Section 4 is devoted to conclusion and discussion. Appendix A explains our notation and convention. In appendix $B$ the metric of the three-parameter $\gamma$-deformed $\operatorname{AdS}_{5} \times \mathrm{S}^{5}$ is rewritten for our convenience. Appendix $\mathrm{C}$ describes in detail the derivation of the metric and the NS-NS two-form from the Yang-Baxter sigma model approach.

\section{Abelian twists of the $\mathrm{AdS}_{5} \times \mathrm{S}^{5}$ superstring}

In this section, after reviewing the formulation of the Yang-Baxter sigma models for the $\mathrm{AdS}_{5} \times \mathrm{S}^{5}$ superstring action with CYBE [48], we consider a particular class of $r$-matrices composed of Cartan generators only. These generate abelian twists, which are examples of the Drinfeld-Reshetikhin twists $[12,13,55]$.

\subsection{Deformed $\mathrm{AdS}_{5} \times \mathrm{S}^{5}$ string actions with CYBE}

We are concerned here with the deformed Green-Schwarz string action [48],

$$
S=-\frac{1}{4}\left(\gamma^{\alpha \beta}-\epsilon^{\alpha \beta}\right) \int_{-\infty}^{\infty} d \tau \int_{0}^{2 \pi} d \sigma \operatorname{Str}\left(A_{\alpha} d \circ \frac{1}{1-\eta R_{g} \circ d} A_{\beta}\right)
$$

where the left-invariant one-form $A_{\alpha}$ is defined as

$$
A_{\alpha} \equiv g^{-1} \partial_{\alpha} g, \quad g \in \mathrm{SU}(2,2 \mid 4) .
$$

Here $\gamma^{\alpha \beta}$ and $\epsilon^{\alpha \beta}$ are the flat metric and the anti-symmetric tensor on the string worldsheet. The operator $R_{g}$ is defined as

$$
R_{g}(X) \equiv g^{-1} R\left(g X g^{-1}\right) g,
$$

where a linear operator $R$ satisfies CYBE rather than mCYBE [36]. Note that the scaling factor $\eta$ may be chosen as $\eta=1$ in our later arguments, due to a peculiarity of CYBE. The $R$ operator is related to the tensorial representation of classical $r$-matrix through

$$
R(X)=\operatorname{Tr}_{2}[r(1 \otimes X)]=\sum_{i} a_{i} \operatorname{Tr}\left(b_{i} X\right) \quad \text { with } \quad r=\sum_{i} a_{i} \otimes b_{i} .
$$

The operator $d$ is given by the following,

$$
d=P_{1}+2 P_{2}-P_{3},
$$


where $P_{i}(i=0,1,2,3)$ are the projections to the $\mathbb{Z}_{4}$-graded components of $\mathfrak{s u}(2,2 \mid 4)$. $P_{0}, P_{2}$ and $P_{1}, P_{3}$ are the projectors to the bosonic and fermionic generators, respectively. In particular, $P_{0}(\mathfrak{s u}(2,2 \mid 4))$ is nothing but $\mathfrak{s o}(1,4) \oplus \mathfrak{s o}(5)$.

For the action (2.1) with a Jordanian R-operator, the Lax pair has been constructed [48] and the classical integrability is ensured in this sense. The $\kappa$-invariance has been proven as well [48]. Here it is worth noting that the nilpotency condition is not necessary for the $\kappa$-invariance and the classical integrability, though it is a sufficient condition to ensure the existence of $1 /\left(1-\eta R_{g} \circ d\right)$. This will be a key observation for our later discussion.

\subsection{Classical R-operators for abelian twists}

In the previous work [48], we have studied classical $r$-matrices of Jordanian type, which satisfy the following properties: 1) solutions of the classical Yang-Baxter equation (CYBE), 2) the skew-symmetricity, 3) the nilpotency. The nilpotency condition is a characteristic property of Jordanian type. A simple example to deform only the $\mathrm{AdS}_{5}$ part [49] is

$$
r_{\text {Jor }}=\frac{1}{\sqrt{2}} E_{24} \wedge\left(E_{22}-E_{44}\right),
$$

where $\left(E_{i j}\right)_{k l} \equiv \delta_{i k} \delta_{j l}$ and the skew-symmetrized symbol $\wedge$ is defined as

$$
a \wedge b \equiv a \otimes b-b \otimes a .
$$

In fact, the associated linear R-operator exhibits the nilpotency $R_{\mathrm{Jor}}^{n}=0$ for $n \geq 3$.

One may adopt "the abelian condition" as the third property, instead of the nilpotency. It is easy to construct such $r$-matrices by using Cartan generators. A typical example is

$$
r_{\text {Abe }}=\sum_{i \neq j} \mu_{i j} h_{i} \wedge h_{j},
$$

where $\mu_{i j}=-\mu_{j i}$ are arbitrary parameters and $h_{i}$ are Cartan generators. We refer the $r$-matrices of this type as to abelian $r$-matrices because these generate abelian twists which are particular examples of the Drinfeld-Reshetikhin twists $[12,13,55]$. These commute with each other and hence satisfy CYBE obviously. Note that abelian $r$-matrices are intrinsic to higher rank cases (rank $\geq 2$ ). For example, for $\mathfrak{s u}(2)$, these become trivial, i.e., $r_{\text {Abe }}=0$. A remarkable point is that the $\kappa$-invariance and the classical integrability are ensured for abelian $r$-matrices, according to the observation denoted in section 2.1 .

\section{$3 \quad \gamma$-deformed $\mathrm{AdS}_{5} \times \mathrm{S}^{5}$ from classical $r$-matrix}

We present here a relation between abelian classical $r$-matrices and $\gamma$-deformed $\operatorname{AdS}_{5} \times \mathrm{S}^{5}$.

\section{$3.1 \gamma$-deformed $\mathrm{AdS}_{5} \times \mathrm{S}^{5}$ with three parameters}

First of all, we give a brief review of gravitational duals of marginal deformations of the $\mathcal{N}=4 \mathrm{SU}(N)$ super Yang-Mills (SYM) theory in four dimensions. 
For a particular class of marginal deformations of $\mathcal{N}=4$ SYM [56] called $\beta$-deformations, the gravitational duals were presented by Lunin and Maldacena [57]. Their original construction is based on an $\mathrm{SL}(2, \mathbb{R})$ symmetry and a single parameter is contained.

Then the solutions were generalized so that three parameters are contained by performing three TsT transformations [58]: 1) $\left.\left(\phi_{1}, \phi_{2}\right)_{\mathrm{TsT}}, 2\right)\left(\phi_{2}, \phi_{3}\right)_{\mathrm{TsT}}$ and 3$)\left(\phi_{3}, \phi_{1}\right)_{\mathrm{TsT}}$. Here $\phi_{i}(i=1,2,3)$ are the Cartan directions in the $S^{5}$ metric and the symbol $\left(\phi_{1}, \phi_{2}\right)_{\mathrm{TsT}}$, for example, means the following. First, a T-duality is performed along $\phi_{1}$. Then $\phi_{2}$ is shifted as $\phi_{2}+\hat{\gamma}_{3} \phi_{1}$ with a constant parameter $\hat{\gamma}_{3}$. Finally a T-duality is taken for $\phi_{1}$ again.

The resulting metric of three-parameter deformed $\mathrm{AdS}_{5} \times \mathrm{S}^{5}$ (in the string frame) and the NS-NS B-field are given by

$$
\begin{aligned}
d s^{2} & =d s_{\mathrm{AdS}_{5}}^{2}+\sum_{i=1}^{3}\left(d \rho_{i}^{2}+G \rho_{i}^{2} d \phi_{i}^{2}\right)+G \rho_{1}^{2} \rho_{2}^{2} \rho_{3}^{2}\left(\sum_{i=1}^{3} \hat{\gamma}_{i} d \phi_{i}\right)^{2} \\
B_{2} & =G\left(\hat{\gamma}_{3} \rho_{1}^{2} \rho_{2}^{2} d \phi_{1} \wedge d \phi_{2}+\hat{\gamma}_{1} \rho_{2}^{2} \rho_{3}^{2} d \phi_{2} \wedge d \phi_{3}+\hat{\gamma}_{2} \rho_{3}^{2} \rho_{1}^{2} d \phi_{3} \wedge d \phi_{1}\right) .
\end{aligned}
$$

Here there is a constraint $\sum_{i=1}^{3} \rho_{i}^{2}=1$ and a scalar function $G$ is defined as

$$
G^{-1} \equiv 1+\hat{\gamma}_{3}^{2} \rho_{1}^{2} \rho_{2}^{2}+\hat{\gamma}_{1}^{2} \rho_{2}^{2} \rho_{3}^{2}+\hat{\gamma}_{2}^{2} \rho_{3}^{2} \rho_{1}^{2} .
$$

For the other field components, see [58]. This solution is often called the three-parameter real $\gamma$-deformed $\operatorname{AdS}_{5} \times \mathrm{S}^{5}$ background. ${ }^{3}$ When $\hat{\gamma}_{1}=\hat{\gamma}_{2}=\hat{\gamma}_{3} \equiv \hat{\gamma}$, the original LuninMaldacena background for the real $\beta$-deformation is reproduced.

In section 3.2, we will present a classical $r$-matrix corresponding to one of the TsT transformations used above.

\subsection{One-parameter case}

As a warm-up, let us consider a simple example of classical $r$-matrix,

$$
r_{\text {Abe }}^{(\mu)}=\mu h_{1} \wedge h_{2} \text {. }
$$

Here $\mu$ is a deformation parameter and the fundamental representation of Cartan generators of $\mathfrak{s u}(4), h_{1}$ and $h_{2}$ are defined as

$$
h_{1} \equiv \operatorname{diag}(-1,1,-1,1), \quad h_{2} \equiv \operatorname{diag}(-1,1,1,-1) .
$$

The action of the associated linear $R_{\text {Abe }}^{(\mu)}$ operator is given by

$$
R_{\text {Abe }}^{(\mu)}\left(h_{1}\right)=-\mu h_{2}, \quad R_{\text {Abe }}^{(\mu)}\left(h_{2}\right)=\mu h_{1}, \quad R_{\text {Abe }}^{(\mu)}(\text { other })=0,
$$

and hence only the $\mathrm{S}^{5}$ part of $\mathrm{AdS}_{5} \times \mathrm{S}^{5}$ is deformed.

Since we are interested in deformations of $S^{5}$, it is convenient to restrict the current $A_{\alpha} \in \mathfrak{s u}(2,2 \mid 4)$ to the $\mathfrak{s u}(4)$ subalgebra as follows:

$$
A_{\alpha}=g^{-1} \partial_{\alpha} g \quad \text { with } \quad g \in \mathrm{SU}(4) / \mathrm{SO}(5) .
$$

\footnotetext{
${ }^{3}$ In [57-59], one can see the classical potential in the corresponding deformed $\mathcal{N}=4$ SYM theory. This potential gets quantum corrections and conformal invariance is broken at quantum level [60, 61]. For this point, we would like to thank J. Fokken, C. Sieg and M. Wilhelm, and D. Giataganas.
} 
With this setup, the $\mathrm{S}^{5}$ part of the classical action (2.1) is reduced to

$$
\begin{aligned}
S & =\int_{-\infty}^{\infty} d \tau \int_{0}^{2 \pi} d \sigma\left(L_{G}+L_{B}\right), \\
L_{G} & =+\frac{1}{2} \gamma^{\alpha \beta} \operatorname{Tr}\left[A_{\alpha} P_{2} \circ \frac{1}{1-2\left[R_{\mathrm{Abe}}^{(\mu)}\right]_{g} \circ P_{2}} A_{\beta}\right], \\
L_{B} & =-\frac{1}{2} \epsilon^{\alpha \beta} \operatorname{Tr}\left[A_{\alpha} P_{2} \circ \frac{1}{1-2\left[R_{\mathrm{Abe}}^{(\mu)}\right]_{g} \circ P_{2}} A_{\beta}\right],
\end{aligned}
$$

where $L_{G}$ is the sigma model part and $L_{B}$ represents the coupling to the NS-NS two-form. Here $\eta$ has been taken as $\eta=1$.

Then the classical Lagrangian given in (3.8) can be rewritten as

$$
\begin{aligned}
L_{G}=-\frac{\gamma^{\alpha \beta}}{2}\left[\partial_{\alpha} r \partial_{\beta} r+\sin ^{2} r \partial_{\alpha} \zeta \partial_{\beta} \zeta+\cos ^{2} r \partial_{\alpha} \phi_{3} \partial_{\beta} \phi_{3}\right. \\
\left.\quad+\frac{\sin ^{2} r}{1+16 \mu^{2} \sin ^{4} r \sin ^{2} 2 \zeta}\left(\cos ^{2} \zeta \partial_{\alpha} \phi_{1} \partial_{\beta} \phi_{1}+\sin ^{2} \zeta \partial_{\alpha} \phi_{2} \partial_{\beta} \phi_{2}\right)\right] \\
L_{B}=\frac{2 \mu \sin ^{4} r \sin ^{2} 2 \zeta}{1+16 \mu^{2} \sin ^{4} r \sin ^{2} 2 \zeta} \epsilon^{\alpha \beta} \partial_{\alpha} \phi_{1} \partial_{\beta} \phi_{2} .
\end{aligned}
$$

For the derivation, see appendix C.

By imposing a parameter relation,

$$
0=\hat{\gamma}_{1}=\hat{\gamma}_{2}, \quad 8 \mu=\hat{\gamma}_{3}
$$

and performing the following coordinate transformation,

$$
\rho_{1}=\sin r \cos \zeta, \quad \rho_{2}=\sin r \sin \zeta, \quad \rho_{3}=\cos r,
$$

we find that the Lagrangian (3.9) and (3.10) are nothing but the ones obtained from the $\gamma$-deformed metric (3.1) and the NS-NS two-form (3.2), respectively. Thus we have shown that the classical $r$-matrix (3.4) corresponds to a TsT transformation $\left(\phi_{1}, \phi_{2}\right)_{\text {TsT }}$.

It would be interesting to reinterpret this result from the viewpoint of a twisted boundary condition by following [62]. In particular, there should be some relation between the classical $r$-matrix and the boundary condition. Along this direction, the correspondence of the Lax pairs would play an important role. The Lax pair constructed in [48] with the $r$-matrix (3.4) should be related to the one constructed in [58], up to a coordinate transformation. Then, it may be possible to find out the relation between the $r$-matrix (3.4) and the twisted boundary condition through the result [62].

\subsection{Three-parameter case}

Now it would be easy to deduce the classical $r$-matrix that corresponds to the threeparameter deformed solution, according to the result obtained in the previous subsection. 
The candidate $r$-matrix is represented by

$$
r_{\mathrm{Abe}}^{\left(\mu_{1}, \mu_{2}, \mu_{3}\right)}=\mu_{3} h_{1} \wedge h_{2}+\mu_{1} h_{2} \wedge h_{3}+\mu_{2} h_{3} \wedge h_{1},
$$

where $\mu_{i}$ and $h_{i}(i=1,2,3)$ are deformation parameters and the Cartan generators of $\mathfrak{s u}(4)$. For $h_{1}$ and $h_{2}$, see (3.5). The remaining $h_{3}$ is defined as

$$
h_{3} \equiv \operatorname{diag}(1,1,-1,-1) .
$$

Then the action of the associated linear $R_{\mathrm{Abe}}^{\left(\mu_{1}, \mu_{2}, \mu_{3}\right)}$ is given by

$$
\begin{array}{ll}
R_{\text {Abe }}^{\left(\mu_{1}, \mu_{2}, \mu_{3}\right)}\left(h_{1}\right)=\mu_{2} h_{3}-\mu_{3} h_{2}, & R_{\text {Abe }}^{\left(\mu_{1}, \mu_{2}, \mu_{3}\right)}\left(h_{2}\right)=\mu_{3} h_{1}-\mu_{1} h_{3}, \\
R_{\text {Abe }}^{\left(\mu_{1}, \mu_{2}, \mu_{3}\right)}\left(h_{3}\right)=\mu_{1} h_{2}-\mu_{2} h_{1}, & R_{\text {Abe }}^{\left(\mu_{1}, \mu_{2}, \mu_{3}\right)}(\text { other })=0 .
\end{array}
$$

With the following parameter identification,

$$
8 \mu_{1}=\hat{\gamma}_{1}, \quad 8 \mu_{2}=\hat{\gamma}_{2}, \quad 8 \mu_{3}=\hat{\gamma}_{3},
$$

and the normalization $\eta=1$, the deformed Lagrangians $L_{G}$ and $L_{B}$ turn out to be

$$
\begin{aligned}
L_{G}=-\frac{\gamma^{\alpha \beta}}{2}[ & \sin ^{2} r \partial_{\alpha} r \partial_{\beta} r+\left(\cos r \sin \zeta \partial_{\alpha} r+\sin r \cos \zeta \partial_{\alpha} \zeta\right)\left(\cos r \sin \zeta \partial_{\beta} r+\sin r \cos \zeta \partial_{\beta} \zeta\right) \\
& +\left(\cos r \cos \zeta \partial_{\alpha} r-\sin r \sin \zeta \partial_{\alpha} \zeta\right)\left(\cos r \cos \zeta \partial_{\beta} r-\sin r \sin \zeta \partial_{\beta} \zeta\right) \\
& +G\left[\sin ^{2} r\left(\cos ^{2} \zeta \partial_{\alpha} \phi_{1} \partial_{\beta} \phi_{1}+\sin ^{2} \zeta \partial_{\alpha} \phi_{2} \partial_{\beta} \phi_{2}\right)+\cos ^{2} r \partial_{\alpha} \phi_{3} \partial_{\beta} \phi_{3}\right. \\
& \left.\left.\quad+\cos ^{2} r \sin ^{4} r \cos ^{2} \zeta \sin ^{2} \zeta\left(\sum_{i} \hat{\gamma}_{i} \partial_{\alpha} \phi_{i}\right)\left(\sum_{j} \hat{\gamma}_{j} \partial_{\beta} \phi_{j}\right)\right]\right] \\
L_{B}=\epsilon^{\alpha \beta} G[ & \hat{\gamma}_{3} \sin ^{4} r \sin ^{2} \zeta \cos ^{2} \zeta \partial_{\alpha} \phi_{1} \partial_{\beta} \phi_{2} \\
& \left.+\sin ^{2} r \cos ^{2} r\left(\hat{\gamma}_{1} \sin ^{2} \zeta \partial_{\alpha} \phi_{2} \partial_{\beta} \phi_{3}+\hat{\gamma}_{2} \cos ^{2} \zeta \partial_{\alpha} \phi_{3} \partial_{\beta} \phi_{1}\right)\right]
\end{aligned}
$$

where the function $G$ is rewritten as

$$
G^{-1}=1+\cos ^{2} r \sin ^{2} r\left(\hat{\gamma}_{1}^{2} \sin ^{2} \zeta+\hat{\gamma}_{2}^{2} \cos ^{2} \zeta\right)+\hat{\gamma}_{3}^{2} \sin ^{4} r \cos ^{2} \zeta \sin ^{2} \zeta
$$

Finally, with the coordinate transformation (3.12), the deformed metric and NS-NS twoform obtained from (3.17) and (3.18) exactly agree with the three parameter $\gamma$-deformed metric (3.1) and (3.2), respectively. The derivation of them is described in detail in appendix C. Thus the classical $r$-matrix (3.13) corresponds to three TsT transformations $\left(\phi_{1}, \phi_{2}\right)_{\mathrm{TsT}},\left(\phi_{2}, \phi_{3}\right)_{\mathrm{TsT}}$ and $\left(\phi_{3}, \phi_{1}\right)_{\mathrm{TsT}}$.

The gravity dual for the real $\beta$-deformation is realized as a particular case with $\hat{\gamma}_{1}=\hat{\gamma}_{2}=\hat{\gamma}_{3}=\hat{\gamma}$. It is wroth noting that complex $\beta$-deformations are argued to yield nonintegrable backgrounds [63]. Probably, there would be no classical $r$-matrix for the complex $\beta$-deformations within the class that allows the Lax pair construction. It may be intriguing to look for the corresponding classical $r$-matrix by admitting that the integrability is broken.

Now the relation between classical $r$-matrices and the $\gamma$-deformed geometries has been clarified. For the $\gamma$-deformed geometries, various things are understood such as the deformed potential in $\mathcal{N}=4 \mathrm{SYM}$ [57-59], the twisted Bethe ansatz [64, 65] and the worldsheet S-matrix [66]. The mirror TBA with twisted boundary conditions is also investigated in $[67,68]$. It would be interesting to argue the relation between them and the classical $r$-matrices used in the Yang-Baxter sigma model approach. 


\begin{tabular}{|l|l|l|}
\hline Operations in SUGRA & Integrable deformations & Classical $r$-matrices \\
\hline TsT transformations & Abelian twists & CYBE, skew-symm., abelian \\
Null Melvin twists & Jordanian twists & CYBE, skew-symm., nilpotent \\
\hline
\end{tabular}

Table 1. Relations among operations in SUGRA, integrable deformations and classical $r$-matrices.

\section{Conclusion and discussion}

In this note, we have considered $\gamma$-deformations of the $\mathrm{AdS}_{5} \times \mathrm{S}^{5}$ superstring as Yang-Baxter sigma models with classical $r$-matrices satisfying CYBE. An essential point is that the classical $r$-matrices are composed of Cartan generators only and generate abelian twists. They do not satisfy the nilpotency condition in comparison to Jordanian deformations considered in [48]. We have presented examples of the $r$-matrices that lead to real $\gamma$-deformations of the $\mathrm{AdS}_{5} \times \mathrm{S}^{5}$ superstring.

Based on our result, one may expect that TsT transformed $\mathrm{AdS}_{5} \times \mathrm{S}^{5}$ geometries could be classified in terms of classical $r$-matrices satisfying CYBE. The conjectured relations are summarized in table 1 , though it is still necessary to make efforts to get supporting evidence. A support is that the type IIB supergravity solution constructed with a Jordanian twist [49] may be regarded as a null Melvin twist, basically following the argument in appendix $\mathrm{C}$ of [69]. We will report on the details in the near future [70]. There are many gravitational solutions obtained as TsT transformed or null Melvin twisted $\mathrm{AdS}_{5} \times \mathrm{S}^{5}$. There should be a classical $r$-matrix for each of them.

At the beginning, the Yang-Baxter sigma model approach has been regarded as a prescription for standard $q$-deformations. Now it seems likely that it potentially contains much broader applications to study integrable deformations. It would provide a guiding principle for classifying possible integrable deformations and the corresponding gravity solutions in terms of solutions of CYBE, which should be called the gravity/CYBE correspondence.

\section{Acknowledgments}

We would like to thank Io Kawaguchi for useful discussions and collaborations at the earlier stage. We are also grateful to thank Sanefumi Moriyama, Ryo Suzuki and Masato Taki for useful discussions. T.M. also thanks Gleb Arutyunov and Riccardo Borsato for useful discussions. T.M. is supported by the Netherlands Organization for Scientific Research (NWO) under the VICI grant 680-47-602. T.M.'s work is also part of the ERC Advanced grant research programme No. 246974, "Supersymmetry: a window to non-perturbative physics" and of the D-ITP consortium, a program of the NWO that is funded by the Dutch Ministry of Education, Culture and Science (OCW).

\section{A Our notation and convention}

Our notation is summarized here. We basically follow the one used in [71]. 
An element of $\mathfrak{s u}(2,2 \mid 4)$ is identified with an $8 \times 8$ supermatrix:

$$
M=\left[\begin{array}{cc}
m & \xi \\
\zeta & n
\end{array}\right] .
$$

Here $m$ and $n$ are $4 \times 4$ matrices with Grassmann even elements, while $\xi$ and $\zeta$ are $4 \times 4$ matrices with Grassmann odd elements. These matrices satisfy a reality condition. Then $m$ and $n$ belong to $\mathfrak{s u}(2,2)=\mathfrak{s o}(2,4)$ and $\mathfrak{s u}(4)=\mathfrak{s o}(6)$, respectively.

In this note we are concerned with deformations of the $S^{5}$ part. Hence it is helpful to prepare an explicit basis of $\mathfrak{s u}(4)$.

Let us first introduce the following $\gamma$ matrices:

$$
\begin{aligned}
\gamma_{1} & =\left[\begin{array}{cccc}
0 & 0 & 0 & -1 \\
0 & 0 & 1 & 0 \\
0 & 1 & 0 & 0 \\
-1 & 0 & 0 & 0
\end{array}\right], \gamma_{2}=\left[\begin{array}{cccc}
0 & 0 & 0 & i \\
0 & 0 & i & 0 \\
0 & -i & 0 & 0 \\
-i & 0 & 0 & 0
\end{array}\right], \gamma_{3}=\left[\begin{array}{llll}
0 & 0 & 1 & 0 \\
0 & 0 & 0 & 1 \\
1 & 0 & 0 & 0 \\
0 & 1 & 0 & 0
\end{array}\right], \\
\gamma_{4} & =\left[\begin{array}{cccc}
0 & 0 & -i & 0 \\
0 & 0 & 0 & i \\
i & 0 & 0 & 0 \\
0 & -i & 0 & 0
\end{array}\right], \gamma_{5}=-\gamma_{1} \gamma_{2} \gamma_{3} \gamma_{4}=\left[\begin{array}{cccc}
1 & 0 & 0 & 0 \\
0 & 1 & 0 & 0 \\
0 & 0 & -1 & 0 \\
0 & 0 & 0 & -1
\end{array}\right] .
\end{aligned}
$$

It is easy to see that

$$
n_{i j}=\frac{1}{4}\left[\gamma_{i}, \gamma_{j}\right] \quad(i, j=1, \ldots, 5)
$$

generate $\mathfrak{s o}(5)$ by using the Clifford algebra

$$
\left\{\gamma_{i}, \gamma_{j}\right\}=2 \delta_{i j}
$$

Note that $\mathfrak{s o}(6)$ is spanned by the set of the generators,

$$
n_{i j}, \quad n_{i 6}=-n_{6 i}=\frac{i}{2} \gamma_{i} .
$$

\section{B Rewriting $\gamma$-deformed backgrounds}

For our purpose, it is convenient to rewrite the metric (3.1) and the NS-NS B-field (3.2) in terms of angle variables;

$$
\rho_{1}=\sin r \cos \zeta, \quad \rho_{2}=\sin r \sin \zeta, \quad \rho_{3}=\cos r .
$$

With the above coordinates, the metric and the NS-NS two-form are given by

$$
\begin{aligned}
d s^{2}= & d s_{\text {AdS }}^{2}+\sin ^{2} r d r^{2}+(\cos r \sin \zeta d r+\sin r \cos \zeta d \zeta)^{2}+(\cos r \cos \zeta d r-\sin r \sin \zeta d \zeta)^{2} \\
+ & G\left[\sin ^{2} r\left(\cos ^{2} \zeta d \phi_{1}^{2}+\sin ^{2} \zeta d \phi_{2}^{2}\right)+\cos ^{2} r d \phi_{3}^{2}\right. \\
& \left.\quad+\cos ^{2} r \sin ^{4} r \cos ^{2} \zeta \sin ^{2} \zeta\left(\sum_{i} \hat{\gamma}_{i} d \phi_{i}\right)^{2}\right], \\
B_{2}=G & {\left[\hat{\gamma}_{3} \sin ^{4} r \sin ^{2} \zeta \cos ^{2} \zeta d \phi_{1} \wedge d \phi_{2}\right.} \\
& \left.+\sin ^{2} r \cos ^{2} r\left(\hat{\gamma}_{1} \sin ^{2} \zeta d \phi_{2} \wedge d \phi_{3}+\hat{\gamma}_{2} \cos ^{2} \zeta d \phi_{3} \wedge d \phi_{1}\right)\right],
\end{aligned}
$$


where the scalar function $G$ is also rewritten as

$$
G^{-1}=1+\cos ^{2} r \sin ^{2} r\left(\hat{\gamma}_{1}^{2} \sin ^{2} \zeta+\hat{\gamma}_{2}^{2} \cos ^{2} \zeta\right)+\hat{\gamma}_{3}^{2} \sin ^{4} r \cos ^{2} \zeta \sin ^{2} \zeta
$$

\section{Derivation of deformed actions}

Here we describe in detail the derivation of the deformed action with the classical $r$ matrix (3.13). The $\mathrm{AdS}_{5}$ part is not deformed and hence we will concentrate on the $S^{5}$ part hereafter.

Let us adopt the following coset parametrization [37]:

$$
g=\Lambda\left(\phi_{1}, \phi_{2}, \phi_{3}\right) \Xi(\zeta) \check{g}_{\mathrm{r}}(r) \quad \in \mathrm{SU}(4) / \mathrm{SO}(5)
$$

with the matrices $\Lambda, \Xi$ and $\check{g}_{\mathrm{r}}$ defined as

$$
\begin{aligned}
\Lambda\left(\phi_{1}, \phi_{2}, \phi_{3}\right) & =\exp \left[\frac{i}{2}\left(\phi_{1} h_{1}+\phi_{2} h_{2}+\phi_{3} h_{3}\right)\right], \\
\Xi(\zeta) & =\left(\begin{array}{cccc}
\cos \frac{\zeta}{2} & \sin \frac{\zeta}{2} & 0 & 0 \\
-\sin \frac{\zeta}{2} & \cos \frac{\zeta}{2} & 0 & 0 \\
0 & 0 & \cos \frac{\zeta}{2}-\sin \frac{\zeta}{2} \\
0 & 0 & \sin \frac{\zeta}{2} & \cos \frac{\zeta}{2}
\end{array}\right), \quad \check{g}_{\mathrm{r}}(r)=\left(\begin{array}{cccc}
\cos \frac{r}{2} & 0 & 0 & i \sin \frac{r}{2} \\
0 & \cos \frac{r}{2} & -i \sin \frac{r}{2} & 0 \\
0 & -i \sin \frac{r}{2} & \cos \frac{r}{2} & 0 \\
i \sin \frac{r}{2} & 0 & 0 & \cos \frac{r}{2}
\end{array}\right),
\end{aligned}
$$

where $h_{i}(i=1,2,3)$ are diagonal matrices given by

$$
h_{1}=\operatorname{diag}(-1,1,-1,1), \quad h_{2}=\operatorname{diag}(-1,1,1-, 1), \quad h_{3}=\operatorname{diag}(1,1,-1,-1) .
$$

These correspond to the Cartan generators of $\mathfrak{s u}(4)$.

With this parametrization, the $S^{5}$ part of the Lagrangian (2.1) can be rewritten as

$$
L=\frac{1}{2}\left(\gamma^{\alpha \beta}-\epsilon^{\alpha \beta}\right) \operatorname{Tr}\left[A_{\alpha} P_{2} \circ \frac{1}{1-2\left[R_{\mathrm{Abe}}^{\left(\mu_{1}, \mu_{2}, \mu_{3}\right)}\right]_{g} \circ P_{2}} A_{\beta}\right],
$$

where $A_{\alpha}=g^{-1} \partial_{\alpha} g$ is restricted to $\mathfrak{s u}(4)$ and the R-operator is defined in (3.15) and we have set that $\eta=1$ in (2.1). For later argument, it is convenient to divide the Lagrangian $L$ into the two parts like $L=L_{G}+L_{B}$, where $L_{G}$ is the metric part and $L_{B}$ is the coupling to the NS-NS two-form, respectively:

$$
\begin{aligned}
L_{G} & \equiv-\frac{1}{2}\left[\operatorname{Tr}\left(A_{\tau} P_{2}\left(J_{\tau}\right)\right)-\operatorname{Tr}\left(A_{\sigma} P_{2}\left(J_{\sigma}\right)\right)\right], \\
L_{B} & \equiv-\frac{1}{2}\left[\operatorname{Tr}\left(A_{\tau} P_{2}\left(J_{\sigma}\right)\right)-\operatorname{Tr}\left(A_{\sigma} P_{2}\left(J_{\tau}\right)\right)\right] .
\end{aligned}
$$

Here the deformed current $J_{\alpha}$ is defined as

$$
J_{\alpha} \equiv \frac{1}{1-2\left[R_{\mathrm{Abe}}^{\left(\mu_{1}, \mu_{2}, \mu_{3}\right)}\right]_{g} \circ P_{2}} A_{\alpha} .
$$

This current contains $\mu_{i}(i=1,2,3)$ and the normalization factor $\eta$. 
To derive the explicit form of the deformed Lagrangian, it is sufficient to compute the projected current $P_{2}\left(J_{\alpha}\right)$ rather than $J_{\alpha}$ itself. Hence the problem is boiled down to solving the following equation,

$$
\left(1-2 P_{2} \circ\left[R_{\mathrm{Abe}}^{\left(\mu_{1}, \mu_{2}, \mu_{3}\right)}\right]_{g}\right) P_{2}\left(J_{\alpha}\right)=P_{2}\left(A_{\alpha}\right)
$$

Note that $P_{2}\left(A_{\alpha}\right)$ is expanded with gamma matrices $\gamma_{i}$ as follows:

$$
P_{2}\left(A_{\alpha}\right)=-\frac{i}{2}\left(\gamma_{1} \partial_{\alpha} r+\sin r\left(\gamma_{2} \cos \zeta \partial_{\alpha} \phi_{1}+\gamma_{3} \partial_{\alpha} \zeta+\gamma_{4} \sin \zeta \partial_{\alpha} \phi_{2}\right)-\gamma_{5} \cos r \partial_{\alpha} \phi_{3}\right)
$$

Then, by combining the expression (C.8) with (C.7), the projected deformed current $P_{2}\left(J_{\alpha}\right)$ can be obtained as

$$
P_{2}\left(J_{\alpha}\right)=\gamma_{1} j_{\alpha}^{1}+\gamma_{2} j_{\alpha}^{2}+\gamma_{3} j_{\alpha}^{3}+\gamma_{4} j_{\alpha}^{4}+\gamma_{5} j_{\alpha}^{5}
$$

with the coefficients

$$
\begin{aligned}
j_{\alpha}^{1}=- & \frac{i}{2} \partial_{\alpha} r \\
j_{\alpha}^{2}=- & \frac{i}{2} \frac{\sin r \cos \zeta}{1+16\left[\mu_{1}^{2} \sin ^{2} 2 \zeta \sin ^{4} r+\left(\mu_{2}^{2} \sin ^{2} \zeta+\mu_{3}^{2} \cos ^{2} \zeta\right) \sin ^{2} 2 r\right]} \\
\times & {\left[\left(1+16 \mu_{2}^{2} \sin ^{2} 2 r \sin ^{2} \zeta\right) \partial_{\alpha} \phi_{1}\right.} \\
& +8\left(\mu_{1}+8 \mu_{2} \mu_{3} \cos ^{2} r\right) \sin ^{2} r \sin ^{2} \zeta \partial_{\alpha} \phi_{2} \\
& \left.+8\left(-\mu_{3}+8 \mu_{1} \mu_{2} \sin ^{2} r \sin ^{2} \zeta\right) \cos ^{2} r \partial_{\alpha} \phi_{3}\right], \\
j_{\alpha}^{3}=- & \frac{i}{2} \partial_{\alpha} \zeta \sin r \\
j_{\alpha}^{4}=- & \frac{i}{2} \frac{\sin r \sin ^{2} \zeta}{1+16\left[\mu_{1}^{2} \sin ^{2} 2 \zeta \sin ^{4} r+\left(\mu_{2}^{2} \sin ^{2} \zeta+\mu_{3}^{2} \cos ^{2} \zeta\right) \sin ^{2} 2 r\right]} \\
\times & {\left[\left(1+16 \mu_{3}^{2} \sin ^{2} 2 r \cos ^{2} \zeta\right) \partial_{\alpha} \phi_{2}\right.} \\
& +8\left(-\mu_{1}+8 \mu_{2} \mu_{3} \cos ^{2} r\right) \sin ^{2} r \cos ^{2} \zeta \partial_{\alpha} \phi_{1} \\
& \left.+8\left(\mu_{2}+8 \mu_{1} \mu_{3} \cos ^{2} \zeta \sin ^{2} r\right) \cos ^{2} r \partial_{\alpha} \phi_{3}\right], \\
& i \\
j_{\alpha}^{5}= & \frac{\cos ^{2} r}{1+16\left[\mu_{1}^{2} \sin ^{2} 2 \zeta \sin ^{4} r+\left(\mu_{2}^{2} \sin ^{2} \zeta+\mu_{3}^{2} \cos ^{2} \zeta\right) \sin ^{2} 2 r\right]} \\
\times & {\left[\left(1+16 \mu_{1}^{2} \sin ^{4} r \sin ^{2} 2 \zeta\right) \partial_{\alpha} \phi_{3}\right.} \\
& +8\left(\mu_{3}+8 \mu_{1} \mu_{2} \sin ^{2} r \sin ^{2} \zeta\right) \sin ^{2} r \cos ^{2} \zeta \partial_{\alpha} \phi_{1} \\
& \left.+8\left(-\mu_{2}+8 \mu_{1} \mu_{3} \cos ^{2} \zeta \sin ^{2} r\right) \sin ^{2} r \sin ^{2} \zeta \partial_{\alpha} \phi_{2}\right] .
\end{aligned}
$$


Finally, $L_{G}$ and $L_{B}$ are given by

$$
\begin{aligned}
L_{G}=-\frac{\gamma^{\alpha \beta}}{2}[ & \sin ^{2} r \partial_{\alpha} r \partial_{\beta} r+\left(\cos r \sin \zeta \partial_{\alpha} r+\sin r \cos \zeta \partial_{\alpha} \zeta\right)\left(\cos r \sin \zeta \partial_{\beta} r+\sin r \cos \zeta \partial_{\beta} \zeta\right) \\
& +\left(\cos r \cos \zeta \partial_{\alpha} r-\sin r \sin \zeta \partial_{\alpha} \zeta\right)\left(\cos r \cos \zeta \partial_{\beta} r-\sin r \sin \zeta \partial_{\beta} \zeta\right) \\
& +G\left[\sin ^{2} r\left(\cos ^{2} \zeta \partial_{\alpha} \phi_{1} \partial_{\beta} \phi_{1}+\sin ^{2} \zeta \partial_{\alpha} \phi_{2} \partial_{\beta} \phi_{2}\right)+\cos ^{2} r \partial_{\alpha} \phi_{3} \partial_{\beta} \phi_{3}\right. \\
& \left.\left.\quad+\cos ^{2} r \sin ^{4} r \cos ^{2} \zeta \sin ^{2} \zeta\left(\sum_{i} \hat{\gamma}_{i} \partial_{\alpha} \phi_{i}\right)\left(\sum_{j} \hat{\gamma}_{j} \partial_{\beta} \phi_{j}\right)\right]\right], \\
L_{B}=\epsilon^{\alpha \beta} G[ & \hat{\gamma}_{3} \sin ^{4} r \sin ^{2} \zeta \cos ^{2} \zeta \partial_{\alpha} \phi_{1} \partial_{\beta} \phi_{2} \\
& \left.+\sin ^{2} r \cos ^{2} r\left(\hat{\gamma}_{1} \sin ^{2} \zeta \partial_{\alpha} \phi_{2} \partial_{\beta} \phi_{3}+\hat{\gamma}_{2} \cos ^{2} \zeta \partial_{\alpha} \phi_{3} \partial_{\beta} \phi_{1}\right)\right] .
\end{aligned}
$$

Here the deformation parameters $\mu_{i}(i=1,2,3)$ in the classical $r$-matrix $(3.13)$ are identified with those of $\gamma$-deformations $\hat{\gamma}_{i}(i=1,2,3)$ through the relations

$$
8 \mu_{i}=\hat{\gamma}_{i} \quad(i=1,2,3) .
$$

Now one can derive the metric and the NS-NS two-form from (C.11) and (C.12). The resulting metric and two-form exactly agree with the metric (B.2) and two-form (B.3) for the three-parameter $\gamma$-deformed $\mathrm{S}^{5}$.

As a particular case, the one-parameter deformed Lagrangian given by (3.9) and (3.10) is given by taking the following parameters:

$$
\mu_{1}=\mu_{2}=0 \quad \text { and } \quad \mu_{3}=\mu .
$$

Open Access. This article is distributed under the terms of the Creative Commons Attribution License (CC-BY 4.0), which permits any use, distribution and reproduction in any medium, provided the original author(s) and source are credited.

\section{References}

[1] J.M. Maldacena, The large- $N$ limit of superconformal field theories and supergravity, Adv. Theor. Math. Phys. 2 (1998) 231 [Int. J. Theor. Phys. 38 (1999) 1113] [hep-th/9711200] [INSPIRE].

[2] S.S. Gubser, I.R. Klebanov and A.M. Polyakov, Gauge theory correlators from noncritical string theory, Phys. Lett. B 428 (1998) 105 [hep-th/9802109] [INSPIRE].

[3] E. Witten, Anti-de Sitter space and holography, Adv. Theor. Math. Phys. 2 (1998) 253 [hep-th/9802150] [INSPIRE].

[4] N. Beisert et al., Review of AdS/CFT Integrability: An Overview, Lett. Math. Phys. 99 (2012) 3 [arXiv:1012.3982] [INSPIRE].

[5] R.R. Metsaev and A.A. Tseytlin, Type IIB superstring action in $A d S_{5} \times S^{5}$ background, Nucl. Phys. B 533 (1998) 109 [hep-th/9805028] [INSPIRE].

[6] I. Bena, J. Polchinski and R. Roiban, Hidden symmetries of the $A d S_{5} \times S^{5}$ superstring, Phys. Rev. D 69 (2004) 046002 [hep-th/0305116] [INSPIRE].

[7] R. Roiban and W. Siegel, Superstrings on $A d S_{5} \times S^{5}$ supertwistor space, JHEP 11 (2000) 024 [hep-th/0010104] [INSPIRE]. 
[8] M. Hatsuda and K. Yoshida, Classical integrability and super Yangian of superstring on $A d S_{5} \times S^{5}$, Adv. Theor. Math. Phys. 9 (2005) 703 [hep-th/0407044] [INSPIRE].

[9] M. Hatsuda and K. Yoshida, Super Yangian of superstring on $A d S_{5} \times S^{5}$ revisited, Adv. Theor. Math. Phys. 15 (2011) 1485 [arXiv:1107.4673] [InSPIRE].

[10] K. Zarembo, Strings on Semisymmetric Superspaces, JHEP 05 (2010) 002 [arXiv: 1003.0465] [INSPIRE].

[11] L. Wulff, Superisometries and integrability of superstrings, arXiv:1402.3122 [INSPIRE].

[12] V.G. Drinfeld, Hopf algebras and the quantum Yang-Baxter equation, Sov. Math. Dokl. 32 (1985) 254 [INSPIRE].

[13] V.G. Drinfeld, Quantum groups, J. Sov. Math. 41 (1988) 898 [InSPIRE].

[14] M. Jimbo, A q difference analog of $\mathrm{U}(g)$ and the Yang-Baxter equation, Lett. Math. Phys. 10 (1985) 63 [INSPIRE].

[15] A. Stolin and P.P. Kulish, New rational solutions of Yang-Baxter equation and deformed Yangians, Czech. J. Phys. 47 (1997) 123 [q-alg/9608011].

[16] P.P. Kulish, V.D. Lyakhovsky and A.I. Mudrov, Extended Jordanian twists for Lie algebras, J. Math. Phys. 40 (1999) 4569 [math/9806014] [InSPIRE].

[17] C. Klimčík, Yang-Baxter $\sigma$-models and dS/AdS T duality, JHEP 12 (2002) 051 [hep-th/0210095] [INSPIRE].

[18] C. Klimčík, On integrability of the Yang-Baxter $\sigma$-model, J. Math. Phys. 50 (2009) 043508 [arXiv: 0802.3518] [INSPIRE].

[19] C. Klimčík, Integrability of the bi-Yang-Baxter $\sigma$-model, arXiv:1402.2105 [INSPIRE].

[20] R. Squellari, Yang-Baxter $\sigma$ model: quantum aspects, Nucl. Phys. B 881 (2014) 502 [arXiv: 1401.3197] [INSPIRE].

[21] S. Schäfer-Nameki, M. Yamazaki and K. Yoshida, Coset Construction for Duals of Non-relativistic CFTs, JHEP 05 (2009) 038 [arXiv:0903.4245] [INSPIRE].

[22] I.V. Cherednik, Relativistically Invariant Quasiclassical Limits of Integrable Two-dimensional Quantum Models, Theor. Math. Phys. 47 (1981) 422 [INSPIRE].

[23] L.D. Faddeev and N.Y. Reshetikhin, Integrability of the Principal Chiral Field Model in (1+1)-dimension, Annals Phys. 167 (1986) 227 [INSPIRE].

[24] J. Balog, P. Forgacs and L. Palla, A two-dimensional integrable axionic $\sigma$-model and $T$ duality, Phys. Lett. B 484 (2000) 367 [hep-th/0004180] [INSPIRE].

[25] N. Mohammedi, On the geometry of classically integrable two-dimensional non-linear $\sigma$-models, Nucl. Phys. B 839 (2010) 420 [arXiv:0806.0550] [InSPIRE].

[26] I. Kawaguchi and K. Yoshida, Hidden Yangian symmetry in $\sigma$-model on squashed sphere, JHEP 11 (2010) 032 [arXiv: 1008.0776] [INSPIRE].

[27] I. Kawaguchi and K. Yoshida, Hybrid classical integrability in squashed $\sigma$-models, Phys. Lett. B 705 (2011) 251 [arXiv:1107. 3662] [INSPIRE].

[28] I. Kawaguchi and K. Yoshida, Hybrid classical integrable structure of squashed $\sigma$-models: a short summary, J. Phys. Conf. Ser. 343 (2012) 012055 [arXiv: 1110.6748] [INSPIRE].

[29] I. Kawaguchi, T. Matsumoto and K. Yoshida, The classical origin of quantum affine algebra in squashed $\sigma$-models, JHEP 04 (2012) 115 [arXiv: 1201.3058] [INSPIRE]. 
[30] I. Kawaguchi, T. Matsumoto and K. Yoshida, On the classical equivalence of monodromy matrices in squashed $\sigma$-model, JHEP 06 (2012) 082 [arXiv: 1203.3400] [INSPIRE].

[31] D. Orlando, S. Reffert and L.I. Uruchurtu, Classical Integrability of the Squashed Three-sphere, Warped $A d S_{3}$ and Schroedinger Spacetime via T-duality, J. Phys. A 44 (2011) 115401 [arXiv:1011.1771] [inSPIRE].

[32] I. Kawaguchi, D. Orlando and K. Yoshida, Yangian symmetry in deformed WZNW models on squashed spheres, Phys. Lett. B 701 (2011) 475 [arXiv:1104.0738] [INSPIRE].

[33] I. Kawaguchi and K. Yoshida, A deformation of quantum affine algebra in squashed WZNW models, arXiv:1311.4696 [INSPIRE].

[34] B. Basso and A. Rej, On the integrability of two-dimensional models with $\mathrm{U}(1) \times \mathrm{SU}(N)$ symmetry, Nucl. Phys. B 866 (2013) 337 [arXiv:1207.0413] [INSPIRE].

[35] F. Delduc, M. Magro and B. Vicedo, On classical q-deformations of integrable $\sigma$-models, JHEP 11 (2013) 192 [arXiv:1308.3581] [INSPIRE].

[36] F. Delduc, M. Magro and B. Vicedo, An integrable deformation of the $A d S_{5} \times S^{5}$ superstring action, Phys. Rev. Lett. 112 (2014) 051601 [arXiv: 1309.5850] [INSPIRE].

[37] G. Arutyunov, R. Borsato and S. Frolov, S-matrix for strings on $\eta$-deformed $A d S_{5} \times S^{5}$, JHEP 04 (2014) 002 [arXiv: 1312.3542] [InSPIRE].

[38] B. Hoare, R. Roiban and A.A. Tseytlin, On deformations of $A d S_{n} \times S^{n}$ supercosets, arXiv: 1403.5517 [INSPIRE].

[39] G. Arutyunov, M. de Leeuw and S.J. van Tongeren, On the exact spectrum and mirror duality of the $\left(A d S_{5} \times S^{5}\right)_{\eta}$ superstring, arXiv:1403.6104 [INSPIRE].

[40] N. Beisert and P. Koroteev, Quantum Deformations of the One-Dimensional Hubbard Model, J. Phys. A 41 (2008) 255204 [arXiv:0802.0777] [InSPIRE].

[41] N. Beisert, W. Galleas and T. Matsumoto, A Quantum Affine Algebra for the Deformed Hubbard Chain, J. Phys. A 45 (2012) 365206 [arXiv:1102.5700] [InSPIRE].

[42] B. Hoare, T.J. Hollowood and J.L. Miramontes, q-Deformation of the $A d S_{5} \times S^{5}$ Superstring S-matrix and its Relativistic Limit, JHEP 03 (2012) 015 [arXiv:1112.4485] [INSPIRE].

[43] B. Hoare, T.J. Hollowood and J.L. Miramontes, Bound States of the q-Deformed $A d S_{5} \times S^{5}$ Superstring S-matrix, JHEP 10 (2012) 076 [arXiv: 1206.0010] [INSPIRE].

[44] B. Hoare, T.J. Hollowood and J.L. Miramontes, Restoring Unitarity in the q-Deformed World-Sheet S-matrix, JHEP 10 (2013) 050 [arXiv:1303.1447] [INSPIRE].

[45] M. de Leeuw, V. Regelskis and A. Torrielli, The Quantum Affine Origin of the AdS/CFT Secret Symmetry, J. Phys. A 45 (2012) 175202 [arXiv:1112.4989] [INSPIRE].

[46] G. Arutyunov, M. de Leeuw and S.J. van Tongeren, The Quantum Deformed Mirror TBA I, JHEP 10 (2012) 090 [arXiv:1208.3478] [INSPIRE].

[47] G. Arutyunov, M. de Leeuw and S.J. van Tongeren, The Quantum Deformed Mirror TBA II, JHEP 02 (2013) 012 [arXiv:1210.8185] [INSPIRE].

[48] I. Kawaguchi, T. Matsumoto and K. Yoshida, Jordanian deformations of the $A d S_{5} \times S^{5}$ superstring, JHEP 04 (2014) 153 [arXiv:1401.4855] [INSPIRE].

[49] I. Kawaguchi, T. Matsumoto and K. Yoshida, A Jordanian deformation of AdS space in type IIB supergravity, arXiv:1402.6147 [INSPIRE].

[50] I. Kawaguchi and K. Yoshida, Classical integrability of Schrödinger $\sigma$-models and q-deformed Poincaré symmetry, JHEP 11 (2011) 094 [arXiv:1109.0872] [INSPIRE]. 
[51] I. Kawaguchi and K. Yoshida, Exotic symmetry and monodromy equivalence in Schrödinger $\sigma$-models, JHEP 02 (2013) 024 [arXiv: 1209.4147] [INSPIRE].

[52] I. Kawaguchi, T. Matsumoto and K. Yoshida, Schroedinger $\sigma$-models and Jordanian twists, JHEP 08 (2013) 013 [arXiv:1305.6556] [INSPIRE].

[53] T. Kameyama and K. Yoshida, String theories on warped AdS backgrounds and integrable deformations of spin chains, JHEP 05 (2013) 146 [arXiv:1304.1286] [INSPIRE].

[54] D. Giataganas and K. Sfetsos, Non-integrability in non-relativistic theories, JHEP 06 (2014) 018 [arXiv: 1403.2703] [INSPIRE].

[55] N. Reshetikhin, Multiparameter quantum groups and twisted quasitriangular Hopf algebras, Lett. Math. Phys. 20 (1990) 331 [inSPIRE].

[56] R.G. Leigh and M.J. Strassler, Exactly marginal operators and duality in four-dimensional $N=1$ supersymmetric gauge theory, Nucl. Phys. B 447 (1995) 95 [hep-th/9503121] [INSPIRE].

[57] O. Lunin and J.M. Maldacena, Deforming field theories with $\mathrm{U}(1) \times \mathrm{U}(1)$ global symmetry and their gravity duals, JHEP 05 (2005) 033 [hep-th/0502086] [INSPIRE].

[58] S. Frolov, Lax pair for strings in Lunin-Maldacena background, JHEP 05 (2005) 069 [hep-th/0503201] [INSPIRE].

[59] D. Berenstein and S.A. Cherkis, Deformations of $N=4$ SYM and integrable spin chain models, Nucl. Phys. B 702 (2004) 49 [hep-th/0405215] [INSPIRE].

[60] J. Fokken, C. Sieg and M. Wilhelm, Non-conformality of $\gamma_{i}$-deformed $\mathcal{N}=4 S Y M$ theory, arXiv: 1308.4420 [INSPIRE].

[61] J. Fokken, C. Sieg and M. Wilhelm, The complete one-loop dilatation operator of planar real beta-deformed $N=4 S Y M$ theory, arXiv:1312.2959 [INSPIRE].

[62] L.F. Alday, G. Arutyunov and S. Frolov, Green-Schwarz strings in TsT-transformed backgrounds, JHEP 06 (2006) 018 [hep-th/0512253] [INSPIRE].

[63] D. Giataganas, L.A. Pando Zayas and K. Zoubos, On Marginal Deformations and Non-Integrability, JHEP 01 (2014) 129 [arXiv:1311.3241] [INSPIRE].

[64] N. Beisert and R. Roiban, Beauty and the twist: the Bethe ansatz for twisted $N=4 S Y M$, JHEP 08 (2005) 039 [hep-th/0505187] [INSPIRE].

[65] C. Ahn, Z. Bajnok, D. Bombardelli and R.I. Nepomechie, Twisted Bethe equations from a twisted S-matrix, JHEP 02 (2011) 027 [arXiv: 1010.3229] [INSPIRE].

[66] C. Ahn, M. Kim and B.-H. Lee, Worldsheet S-matrix of beta-deformed SYM, Phys. Lett. B 719 (2013) 458 [arXiv:1211.4506] [INSPIRE].

[67] G. Arutyunov, M. de Leeuw and S.J. van Tongeren, Twisting the Mirror TBA, JHEP 02 (2011) 025 [arXiv: 1009.4118] [INSPIRE].

[68] S.J. van Tongeren, Integrability of the $A d S_{5} \times S^{5}$ superstring and its deformations, arXiv: 1310.4854 [INSPIRE].

[69] V.E. Hubeny, M. Rangamani and S.F. Ross, Causal structures and holography, JHEP 07 (2005) 037 [hep-th/0504034] [INSPIRE].

[70] T. Matsumoto and K. Yoshida, Jordanian deformed $A d S_{5} \times S^{5}$ backgrounds from TsT-transformations, in preparation.

[71] G. Arutyunov and S. Frolov, Foundations of the $A d S_{5} \times S^{5}$ Superstring. Part I, J. Phys. A 42 (2009) 254003 [arXiv:0901.4937] [INSPIRE]. 